\title{
Watershed Algorithm for PCB Automatic Optical Inspection Systems
}

\author{
PingFeng ${ }^{1,}$,, Qi-yuan Gong ${ }^{2,}$ b $^{*}$,Tao Cheng $^{3, \text { c }}$ \\ ${ }^{1,2,3}$ College of Mechatronics and Control Engineering, Shenzhen University, Shenzhen Guangdong \\ 518060, P.R. China

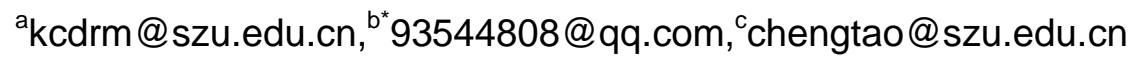

\begin{abstract}
Keywords:Machine Vision; Watershed Algorithm; Region Segmentation; Local Extreme; Defect Recognition

Abstract. The production of the PCB board is related to the development of the electronics industry. In order to improve the quality of the circuit board, Startingfrom the detection of single PCB board and replacing the traditional manual inspection mode through using the methods of machine vision is to improve the efficiency. For "the outer layer inspection" link of the PCB substrate in the generalized processing,the use of machine vision detection algorithm based on watershed algorithm and the integration of organic into existing automated optical inspection equipment not only ensureshigh efficiency but also reduces the artificial false detection rate through replacing the human eye with the use of the machine vision. Different from traditional detection algorithm, the advantages of watershed algorithm are mainly reflected in the extractingsegmentation target area pixel value and regions by calculation and comparison of local extreme position to identify deficiencies and give the type of defect identification purposes. Experimental results show that the use of this method is effective to improve the efficiency and accuracy of defect recognition.
\end{abstract}

\section{Introduction}

PCB (Printed Circuit Board) is a substrate connecting and printing of the formed original design according to the predetermined points between the common substratesprinting plates. What the main function is to make a variety of electronic components form a predetermined circuit connection, acting as a relay transmission, that is the key to the electronic interconnect products. Printed circuit board manufacturing qualitynot only directly affects the reliability of electronic products, but also affects the overall competitiveness of the system products.

Currently, the global PCB industry, electronic components industries' output accounted for one-quarter of GDP or more in each of the electronic components industry is the largest proportion of the industry, also of contemporary electronic components industry in the most active industry.PCB industryenjoys a unique position in the electronics industry base. According to market research firm NT Information statistics, the global PCB industry value in 2012 was \$-59.796 billion. The agency forecaststhe global PCB output value of 2013was 598.08 billion US dollars, the gradual improvement in the global economy, driven by the PCB in 2014 indicates that GDP will grow by 3.84 percent, to $\$-62.102$ billion.

Now the methods of the detection of the PCB board include manual testing and automated testing, also it's obvious that the advantages of the automatic detection, high speed and high efficiency, are much better than the others.

Since the early 1980s, the international have begun to put a lot of financial, material and human resources on developing PCB automatic optic inspecting system. Currently, in order to research AOI system, the mature foreign companies, such as Israeli Orbotech, Camtek, British Teradyne, Korea Samsung and Japan Ormon, have developed their own technological products.

The Israel Orbotech company's Spiron-8800AVIP is the first automatic execution defect detecting product in the world and it's set up by the methods of the use of design rules, the feature 
point comparison method, IPSO technology combined contour to detect the flaws such as open circuit, short circuit, the minimum line width, gap, highlights, depression and residual copper etc. It can detect the line width and line from the specification of 1.5-10 mile (38-250um). The maximum detection area is $686^{*} 1448 \mathrm{~mm}$. The speed of detection is $160 \mathrm{pc} / \mathrm{h}$ if the reference panel size is $457 * 610 \mathrm{~mm}$ and the width is $6-4 \mathrm{mile}(150-100 \mathrm{um})$. Also when the line width is 3 mile (75um), the speed is $120 \mathrm{pc} / \mathrm{h}$. South Korean Samsung company VSS-3C type of advanced three-dimensional visual detector, with superior optic detection performance, the chip can be installed, three-dimensional welding state and the bent pin automatic inspection. It is equipped with high-resolution digital camera, color image analysis and automatic intelligent programming system, an optional resolution of $15 \sim 20 \mathrm{um}$ and speed can be $16 \mathrm{~cm} / \mathrm{s}$. The subtraction algorithm using in the operation is the main trend in the AOI system, but with the development of the efficiency can't satisfied the need of the industrial standard. With analysis the algorithm that we can find the further developmental space about the efficiency, and it is necessary to find a better algorithm applying to the system.

Watershed algorithm can be classified as an image segmentation method based on region. The concept of the topography ${ }^{[1]}$ is this: if there water flows into a depression, watershed divides the inflow of water in the region as the dividing line. More intuitive concept is to assume the existence of such a lake terrain: depression is its local minimum. These depressions will be injected with water and slowly filled. Different water will meet the rising swale, the establishment of a dam in the place of encounter, until the water flooded the highest point of the terrain, the end. This established a division of the various regions of the watershed, or called watershed line. Currently, the watershed algorithm can be roughly divided into two types, one is based on mathematical morphology algorithm definition by Vincent and Soille ${ }^{[2]}$ proposed; The other is based on the algorithm from the topographical definition by Meyer and Fernand ${ }^{[3]}$ suggested that the algorithm is sensitive at the faint edges and you can get a single pixel wide, connected, closed contour and accurate location.

There have never been that the watershed algorithm was used in the PCB defect detecting system. Watershed algorithm has a variety of implementations, including token-based watershed transform and Euclidean distance mapping method. The essence of those is distance transforming and divides the threshold required in order to achieve regional segmentation. Considering of this we contact with the line width transformation, calculating the line width of the regions and setting the threshold, in order to find the line defect those are classified and marked automatically. This paperintroduces the correlational algorithm principles and application in the AOI system.

The remainder of the paper is organized as follows. First, we review the literature on watershed algorithm theory and the achievements we have made including the process of the experiment and the results. Nextwe provide an introduction about the hardware and software that we have made. Then, the experimental results and analysis with our new proposed method are presented and contrast with the original method. Finally, a computational comparison of the solution approaches is presented followed by concluding remarks and suggestions for future research in this area.

\section{Detecting Method Based on Watershed Algorithm}

\section{Watershed Algorithm}

Watershed algorithm ${ }^{[4]}$ can extract the goal for closed boundary with the single pixel width quickly, it has been widely used in the split technology and flexible applied to the automatic optic inspection, but it is sensitive to the noise and gradient-dependent. Low-contrast image is so easy to lose important contours and causes the segmentation problem that generating a large number of false edges in the process of image segmentation and that seriously interfere with the identification 
of the target's edge. Currently the methods to solve the problemof over-segmentation are divided into two categories. The first one is to split after some combinational rules which determine the over-segmentation regions combination and this rule will become more complexwith the growth of the number of those regions, wasting so much time. The other way is pretreated that it is based on the marker extraction, through extracting the target area of an image previously to regulate the final number of the zones, and the key point is how to get the effective way focusing on the selected area markers. The project will start from PCB's characteristics of the defect detecting with integrated survey in the calculation of topographical distance algorithm, regional extremum search, morphology micro areas extracted or filtered, ridges of translation and rotation, self-adaptive extracting the aspects of the two ways in the ridge height anomaly, in order to find suitable methods, and testthe improvement and optimization through experimenting on a various types of PCB.

\section{Experimental Achievement}

The project is based on the watershed transformation mainly using the theory of the mathematical morphology to construct the detecting algorithm of PCB circuit board with preventing the undetected and reducing the false alarms to improve the accuracy, achieve accurate, stable and applicability of standards. First of all we need to obtain a high quality image by the image acquisition unit. Then we take a pretreatment of an image through mathematical morphology algorithm and then take it to the defect identification module to make detecting and automatic identifying the defect location. The statistical data of the corresponding output information is reserved to facilitate future repair. Line defect detecting technique is as shown in Fig.1.

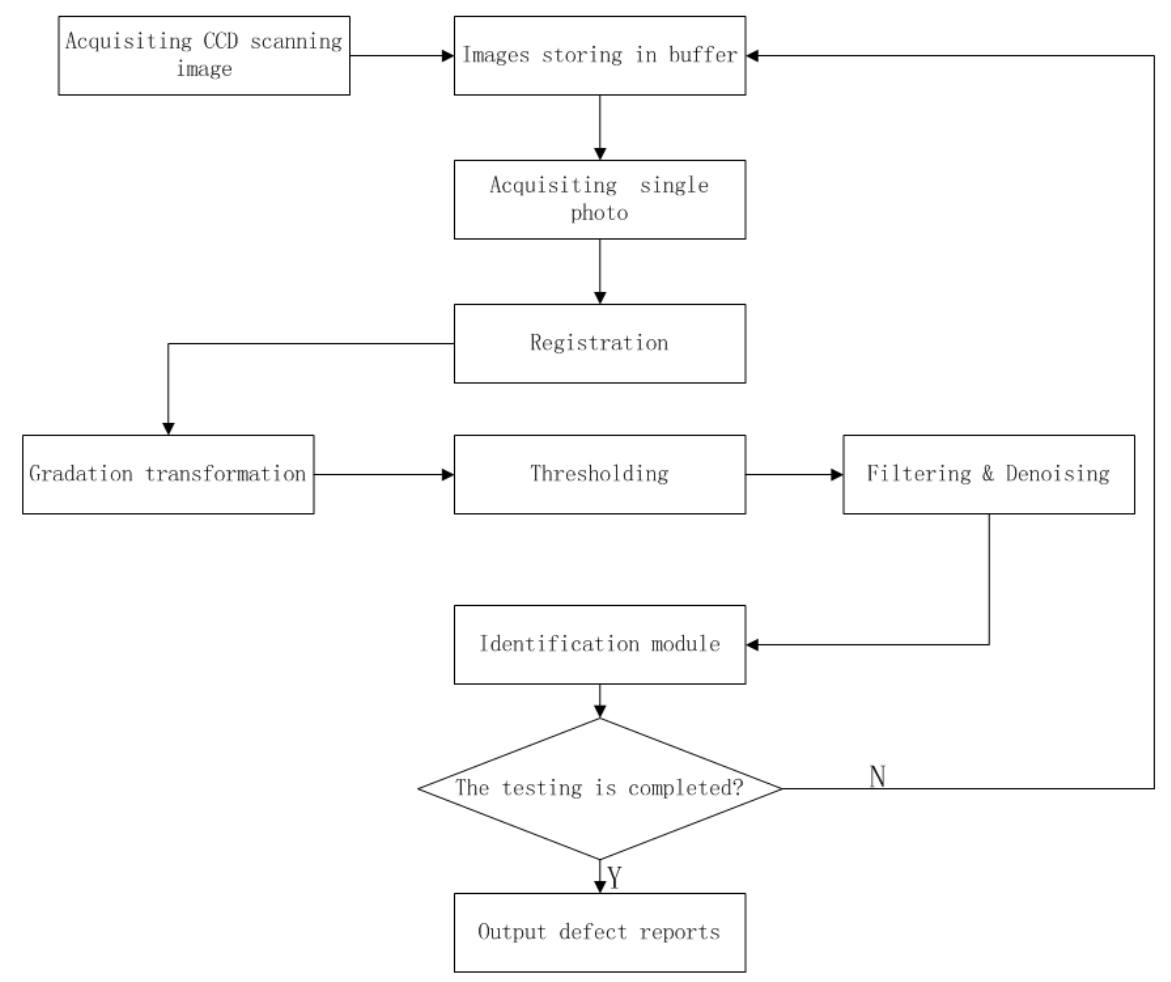

Fig.1PCB defect detection technological roadmap

Gerber file $^{[5]}$ format is the subset of RS-274D of IEA standard and it can be generated by all the PCB-CAD systems, also it can be compatible in all the plotters. According to Gerber data unit, typically inches, and scanning accuracy, it is easy to determine the correspondence between the Gerber data including pixels and the standard pictures unit data and then we can get the standard pictures which come from analyzing and matching scanning image, as shown in Fig.2. 


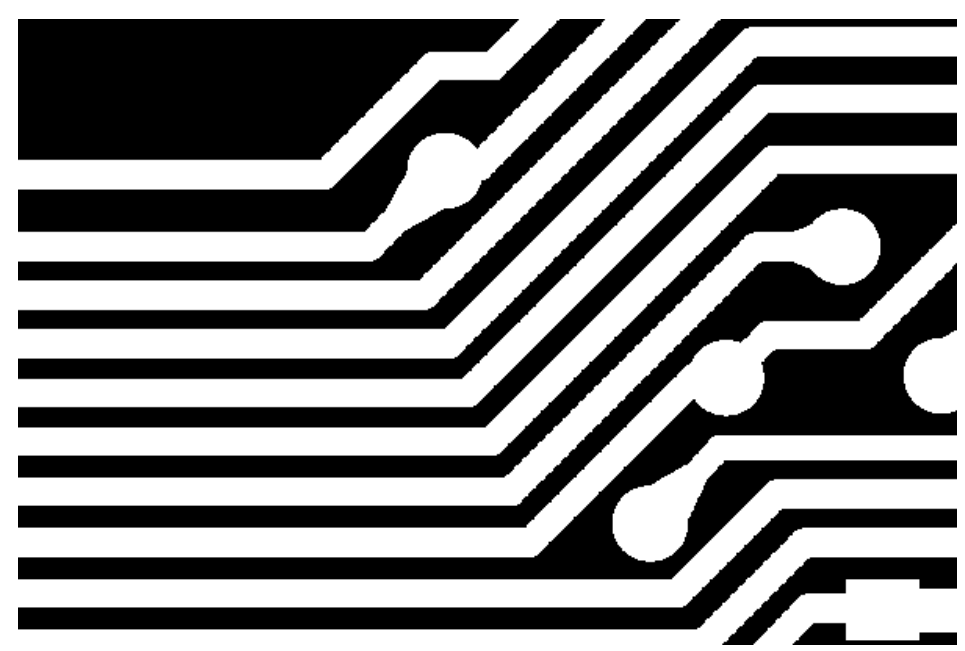

Fig.2 Obtain standard image IO from analyzing the Gerber file (area of $600 * 400$ pixels, taken from the original $45000 * 25000$ pixels standard image, Black: a substrate region, White: copper area)

Next it is important to search for the watershed pixels in the standard image. The calculation of the image search what is so called watershed transformation is very large. The configuration includes Intel dual-core processor i3-3220 (3.30GHz), integrated graphics processor, Matlab 8.2 running on the computer with $8 \mathrm{~GB}$ memory so that the time of processing the original pixels of image with $45000 * 25000$ is 5.14 seconds and the image includes 60 sub-regions with the $3000 * 6592$ pixels. So it is necessary to improve the efficiency and the time of the algorithm of us has reduced to 1.88 seconds in the same environment.

It should be noted that we not only calculate the copper regions watershed pixels to detect the defect about disconnection and gap (rat bite) etc. Also we must calculate the watershed pixels of the substrate area to identify the defect of short circuit and spursetc. Fig. 3 shows the results of the watershed transformation of standard image.

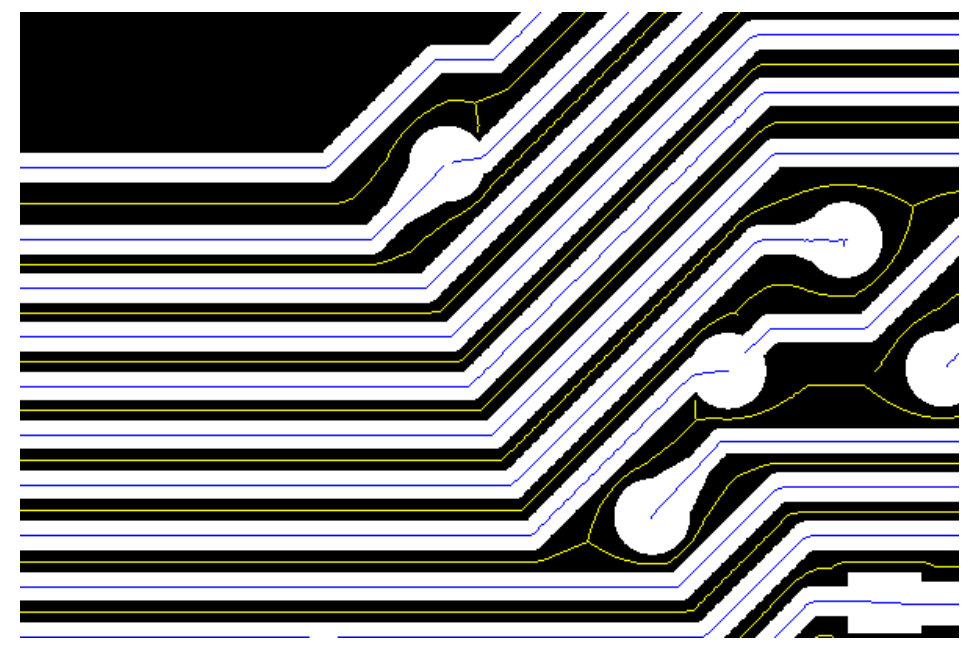

Figure.3 Standard image and watershed transformation results (Black: base area, White: copper area, Yellow: the watershed pixels of base area, Blue: watershed pixels of copper area)

The use of automated optical inspection equipment is to scan for the batch of plate optimized path planning for PCB scanning, through array camera automatically adjusting the light source of illumination and uniformity of compensation and then making thresholding and image mosaic to get the scanning image. Next we must search the four corners of PCB positioning mark on a scanned image and get the two-match transformation coefficients which comes from the coordinates of standard image and the scanned image through the contrasting the scanned four marks with the 
Gerber file, and that is the image registration. Then according to the registration relation, we put the watershed pixels of the standard image mapping onto the scanned image, and the theory of the geometry is to make the relevant translation and rotation of the standard image watershed pixels overlaying on the scanned image in order to form the characteristic pixels in Fig.4.

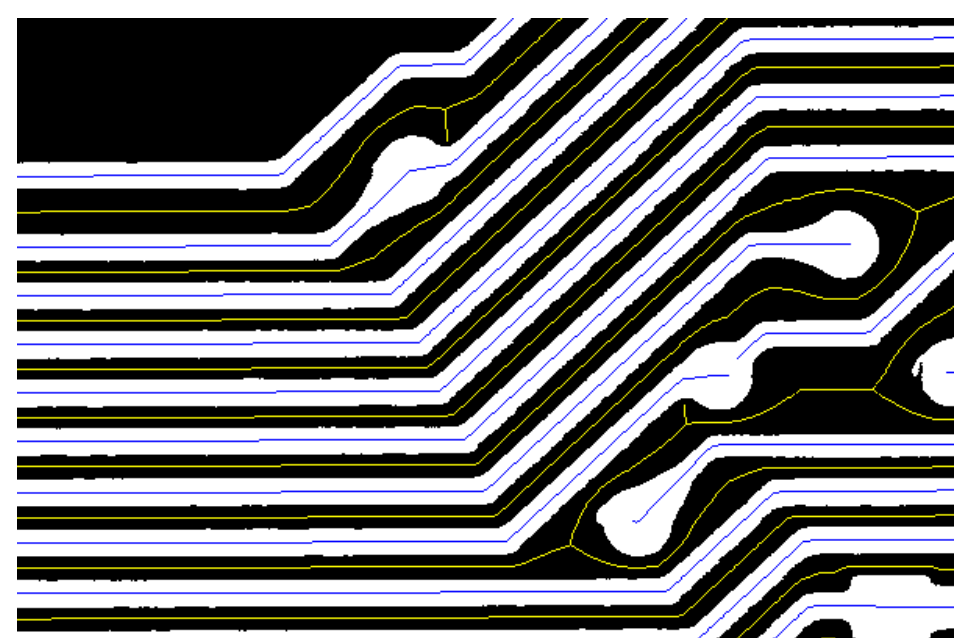

Fig.4the scanned image and the characteristic pixels the watershed pixels of the standard image through the registration bilinear transform what is called the translation and rotation become the characteristic pixels. Black: base area, White: copper area, Yellow: characteristic pixels of the base area, Blue: characteristic pixels of the copper area)

Through contrasting the elevation of the watershed pixels and the relevant characteristic pixels and the height is the shortest distance to the nearest another type region. If the difference between the distance and the standard is greater than a given threshold, then we identify it as defect.Only through detecting of defects in the surrounding area after the noise filter and then calculating the elevation difference can we find the special regions that the difference is so large and we can identify these are the defect goals. The method not only reduces the false detection rate, but also reduces the amount of computation.

Finally, we take the locations of the defects and the type information about the defects to output a report that can be used to track and fix the defects.

\section{Experimental Configuration}

\section{Software}

\section{Design and Implementation of a Watershed for PCB Defect Detection Algorithm}

In the entire complex PCB production process, with external and human factors, resulting in a lot of quality problems such as short circuit, bumps, depressions, residual copper, pinholes, drain lines, drain drilling, etching over etching is insufficient, wrong size and breaking the hole, that it has make a serious influence on the operation process.

The project will study various existing watershed algorithm, using massive data base of existing work was to identify or obtain improved type of algorithm for various defects, to prevent false negative and,build with matching the computer hardware environment and a satisfactory processing speed.

\section{Integration with Online Testing Algorithm}

Common image processing software for image size is limited, such as the most widely used Photoshop image Processing software, the latest version supports a maximum image size of 30000*30000 pixels and the computer is configured with demanding, and typical PCB image size 
$45000 * 25000$ pixels, the image of such a big deal directly is impractical, so the algorithm is integrated into automated optical inspection equipment division after the first process is the sub-region. Sub-region can't be too large, otherwise the computer memory and CPU pressure is too large, easy to read and write frequently lead to crashes (greatly extended processing time). On the other hand, watershed algorithm in image edge is often not the correct result, thus requiring association the sub-region have overlapping portions, if the sub-region is too small, it will greatly increase the number of pixels involved in the calculation, reducing efficiency.

Online tests on the process also involves registering the scanned image registration and Gerber files registration, the source of illumination and uniformity of compensation, linear array camera automatically adjusting, the scan path planning, image preprocessing, image stitching, coordinate conversion and so on. Module should be analyzed by the overall performance of the algorithm, the time complexity of algorithm analysis, evaluation of the compatibility and the use of constructed sequence of these modules is reasonable.

\section{Hardware}

For experimental hardware AOI (Automatic Optic Inspection), we have made a number of the system function set: Image quality, scan speed, light illumination compensation, the image gradation compensation, image stitching, image registration, image positioning, defect detection; performance tests include: Gerber file parsing efficiency, efficiency of image stitching, image registration efficiency, detection efficiency; reliability tests include: the false detection rate, undetected rate, the program is stable; operability tests include: man-machine interface operation, the light source control operation, camera control operation.

The main results achieved are:

(1) Based on a standard PCB Gerber file images quickly produce this project through self-designed analytical Gerber file sub-module applies to the main program to quickly generate images via standard PCB Gerber file. It can be dynamic, comprehensive presentation of its information characteristics, parsed image with rich information, not omission, portability, and without considering other factors, strong anti-interference ability to generate PCB picture time is short, high efficiency.

(2) Large-format image-based self-learning quick scan planning methods. Large format images quickly scan planning technology roadmap is divided into scanning direction and non-scanning direction of two parts: $\mathrm{X}$-axis motion planning (linear array camera image acquisition direction of motion planning, Fig.5) and Y-axis direction motion planning (linear array camera does not work at this stage, but the Y-axis positioning accuracy required to compensate, Fig.6). Fig. 7 shows the real object

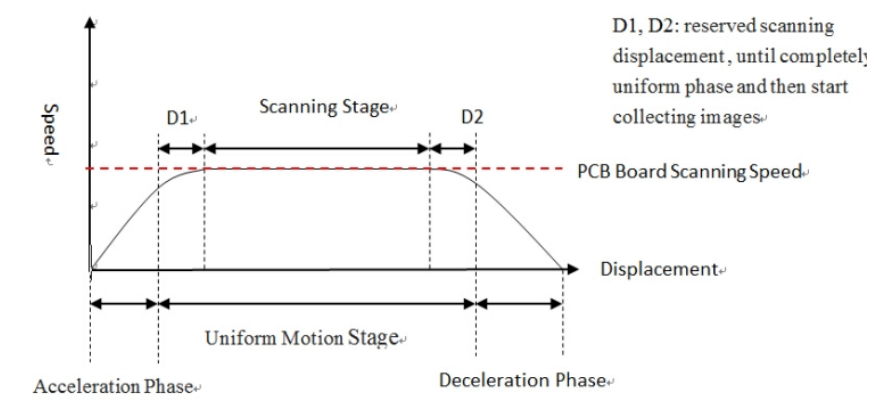

Fig. $\quad 5 x$-axis motion planning

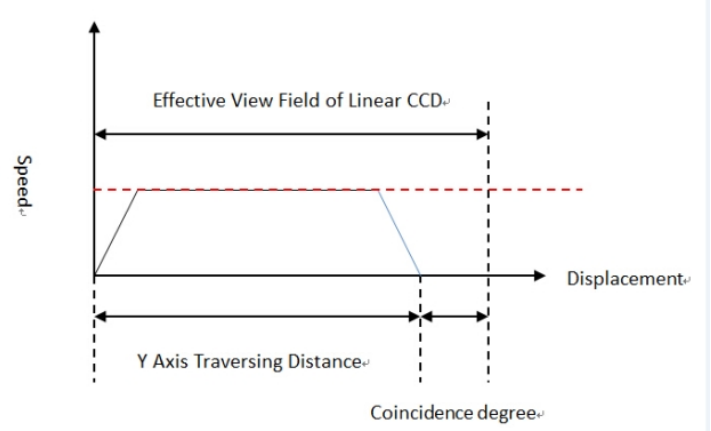

Coincidence degree

Fig. $6 y$-axis motion planning 


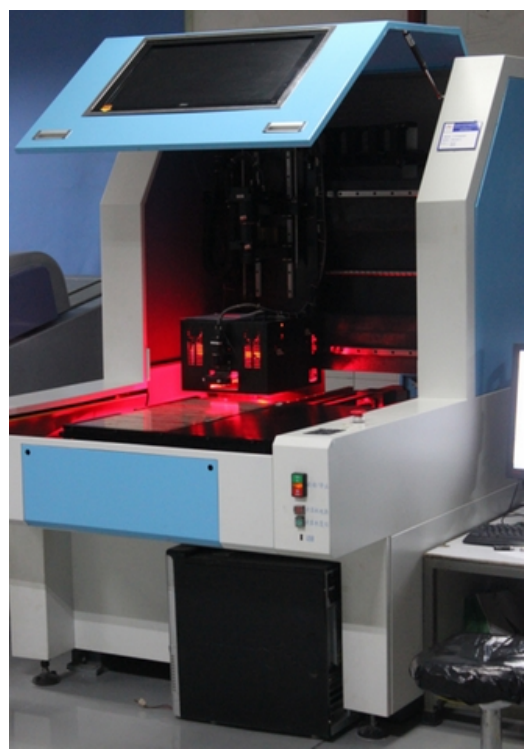

Fig. 7 Automatic detection hardware

(3) The structure of the light source of illumination, uniformity tests and compensation. Since linear CCD camera exposure time can only take a linear portion of the object, so the light source or the camera itself is the cause of intensity inhomogeneity can only occur in the scanning column by standard gray plate scanned image, the grayscale value computed column direction as the calibration standard. Uniformity compensation algorithm is as follows: take the standard gray plate scan each column as the maximum average gray image uniformity correction reference value $\mathrm{G}_{\mathrm{s}}$, and then take the gray board standard scan any column gray average gray value $\mathrm{G}_{\mathrm{s}}(\mathrm{i})$, any column calculated gradation correction coefficient $\mathrm{K}(\mathrm{i})$.

$$
\mathrm{K}(\mathrm{i})=\mathrm{G}_{\mathrm{s}} / \mathrm{G}_{\mathrm{s}}(\mathrm{i})
$$

It determined according to the gradation correction coefficient can be obtained scanned image gray compensated value $G^{\prime}(i)$.

$$
\mathrm{G}^{\prime}(\mathrm{i})=\mathrm{K}(\mathrm{i}) \mathrm{G}(\mathrm{i})
$$

$\mathrm{G}(\mathrm{i})$ is the gray value of the scanned image before correction.

\section{Experimental Results and Analysis}

Image search criteria "watershed" pixels (ie picture watershed transform) calculation is very large, using the configuration Intel dual-core processor i3-3220 (3.30GHz), integrated graphics processor, running Matlab 8.2 $2^{[6]}$ on 8GB DDR3-1600 memory computer.The watershed command processing $3000 * 6592$ pixels image takes 5.14 seconds (the original $45000 * 25000$ pixels standard image contains 60 sub-regions whose contains $3000^{*} 6592$ pixels) and it is necessary to take measures to improve the computational efficiency, a new algorithm which is currently built in the same environment to reduce the computation time from 5.14 seconds to 1.88 seconds, which is a good start.

It should be noted that, not only to calculated pixel copper watershed area for disconnection, gap (rat bite), and other defect detection, also calculate pixel substrate watershed area for defect detection for example short projecting copper (spurs) etc. Fig.8 shows the results of the standard image of a watershed transformation. 


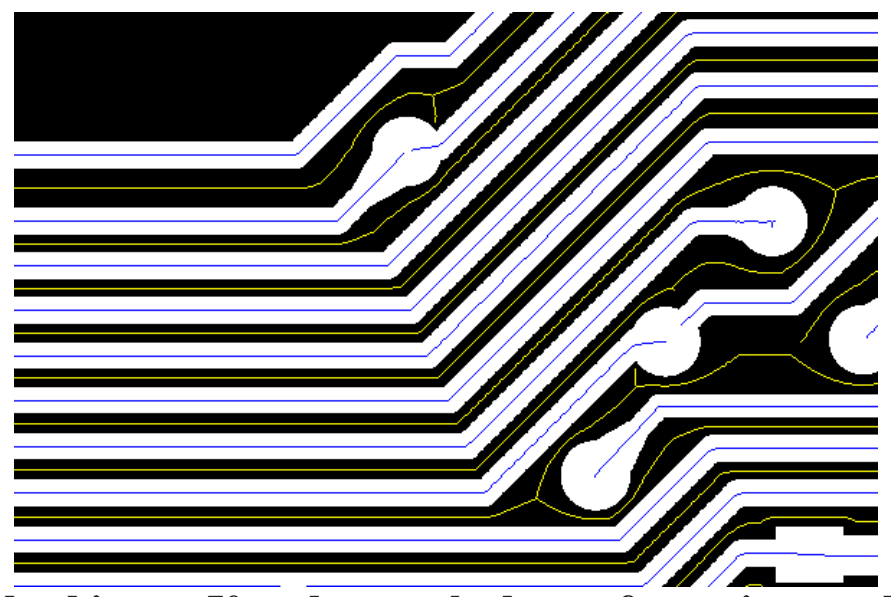

Fig.8Standard image I0 and watershed transformation results (Black: a substrate region, White: copper area, Yellow: a pixel substrate watershed area, Blue: copper area watershed pixels)

Fig. 8 shows the results (first standard image were calculated watershed pixels of copper area and the base area of application of the new algorithm, then matched quasi- scanned image and the standard image coordinate transformation between the two stars, the standard image watershed pixel coordinate transformation feature to scan an image pixel coordinates, and then comparative the distance characteristics one by one from the value of the pixel between the present pixels and the corresponding pixels, if the difference is greater than a given threshold, then the noise impact assessment determines that should be defective), visible short circuit, copper sudden, no one missed gap, does not affect the electrical properties of different (mostly error caused by the optical system) are no false positive. It may be initially determined that the new algorithm is stable, reliable and efficient.

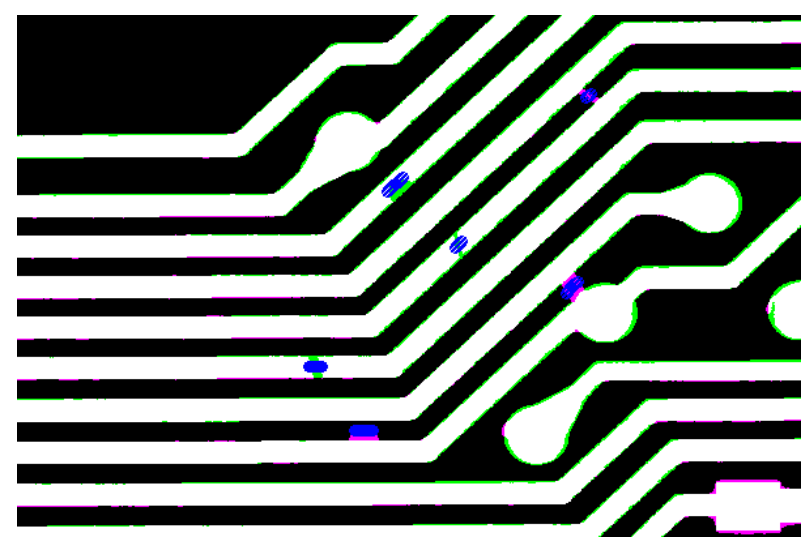

Fig. 9fter implementation of the new standard image I0 watershed algorithm analysis test results obtained by scanning the image I (Black: I and I0 copper area, White: I and I0 copper area, White: I deleted region copper, Purple: I cover copper surplus area, Blue: defect identification area)

\section{Conclusion}

The production of the PCB board is related to the development of the electronics industry and the automatic inspecting is the most important step during the production process.Currently the most of the automatic optic inspection equipment adopt subtraction ${ }^{[7]}$ between the standard image and the testing image, but only few can get the accurate matching templates with the high level of false positive and unsatisfied precision. Considering the traditional method applying in the PCB automatic optic inspecting system, the method which has never been used in this area, based on the watershed algorithm applying in the automatic optic inspecting has made a huge improvement on 
efficiency in case of preventing false positive and false negative. With a large number ofexperiments we have made that the hardware and software systems indicate the method is fit for the inspecting and with the future debugging the result will meet our demands. In the further development the appliance with the watershed algorithm will appear in any other machine vision inspecting areas.

\section{Reference}

[1]J.Serra, Image Analysis and Mathematical Morphology, Academic Press, New York, 1982.

[2] L Vincent, P Soille, Watersheds in digital spaces: An efficient algorithm based on immersion simulations (1991),IEEE Trans Patt Anal Mach Intell, 13(1991) 583-598.

[3] Meyer, Fernand, Topographic distance and watershed lines, Signal Processing, 38.94(1994)113-125.

[4] Bieniek A, Moga A. An efficient watershed algorithm based on connected components[J]. Pattern Recognition, 2000, 33(99):907-916.

[5] Yao J, Yu-Tang Y E, Zhang J, et al. Research of parsing Gerber file in PCB automatic optical inspection[J]. Computer Engineering \& Design, 2012, 33(6):2481-2485.

[6] Gonzalez R C, Woods R E, Eddins S L. Digital image processing using MATLAB[J]. Digital Image Processing Using Matlab, 2004, 21(84):197-199.

[7] Chauhan A P S, Bhardwaj S C. Detection of Bare PCB Defects by Image Subtraction Method using Machine Vision[J]. Lecture Notes in Engineering \& Computer Science, 2011, 2191(1). 